\title{
Development of Fish type Robot based on the Analysis of Swimming Motion of Bluefin Tuna -Experimental Discussion-
}

\author{
Katsuya Kugai ${ }^{\text {a, }}$ and Ikushi Yamamoto ${ }^{a}$ and Yuuki Matsumoto ${ }^{\text {a }}$ and Meitarou Takahashi ${ }^{\text {a }}$ \\ ${ }^{a}$ KINDAI University Technical College, Japan
}

\begin{abstract}
The swimming motion of Tuna type fishes has excellent ability in its speed and efficiency. On the other hand, some studies on the most efficient swimming motion have been reported using numerical analysis of a two-point hinge mechanism model. However, since most conventional fish robots hold their caudal fin in a spring, the caudal fin works only passively and can not confirm theoretical results in an experimental way. Therefore, we developed a fish type robot that combines caudal fin angle actuating mechanism and tail oscillating mechanism. Using this robot, we experimentally investigated the change in swimming speed due to the difference in swimming motion.
\end{abstract}

Index Terms - Caudal fin, Lift and Drag, Fish type robot, Propulsive force

\section{INTRODUCTION}

Swimming with a fin has some great features compared to a screw. The fins do not cause the submarine to get stuck, as it does with the seaweed like a screw. And swimming by the fins will hardly lift up the sediment on the seabed and disturb the view.

However, propulsion with fins does not seem to be more effective than screws in speed and energy efficiency. So, we focused on the excellent swimming ability of bluefin tuna. The maximum speed of bluefin tuna is estimated to be $80 \mathrm{~km} / \mathrm{h}$ and swims over 3000 $\mathrm{km}$ to and from the Pacific Ocean [1].

The Calangiform swimming of tuna and dolphins is modeled by a two-joint bending mechanism by Azuma [2]. Lighthill has applied a two-dimensional linearized wing theory towards a two-joint bending mechanism to provide an accurate analysis of tuna swimming ability. Nakajima and Ono described an analysis method combining the slender body theory and the linearization wing theory. Then, it was reported that the propulsion efficiency is the best when the feathering parameter $\theta=$ 0.8. [3][4]

\footnotetext{
* Corresponding author: 7-1, Kasugaoka, Nabari-city, Mie-pref.,
} 518-0459, Japan, kugai@ktc.ac.jp
However, in that study, the motion of the fin angle is limited by a sine wave. According to the results of our motion analysis using real bluefin tuna, the motion of the fin angle looks like an arctangent waveform [5]. Therefore, as a result of investigation using a simple wing theory, we propose $\theta \mathrm{f} / \theta \mathrm{w}=0.85$ as the motion of the caudal fin to obtain the most effective propulsion efficiency [6]. Here, $\theta \mathrm{f}$ is a caudal angle, and $\theta \mathrm{w}$ is a water inlet angle to the caudal fin.

In order to confirm these theoretical suggestions by experiments, we have developed a tuna-type robot that can independently control tail oscillation and caudal fin angle. This robot can reproduce arbitrary 2 -joint bending motion with software. However, it is difficult to achieve periodic caudal fin motion without angular decay or phase lag.

Therefore, in this paper, we describe a system that can move the caudal fin correctly. Then, using this robot, we experimented to move the caudal fin under several control methods and compare swimming speeds. As a result, it was found that the maximum velocity can not be obtained in the operation of $\theta=0.8$ and the operation of $\theta \mathrm{f} / \theta \mathrm{w}=0.85$. We will explain this at the end of this paper.

\section{METHODS}

\section{A. Development of Tuna Type Robot}

We developed a tuna type robot shown in Fig.1. To oscillate the tail, a scotch yoke mechanism is employed to convert the rotational motion of the motor into an oscillating motion of the tail. This mechanism can produce an accurate sine waveform. The oscillation amplitude is fixed at $\pm 0.04 \mathrm{~m}$, but the frequency can be changed by the motor speed. A parallel link mechanism is adopted to change the angle of the caudal fin. The angle of the caudal fin changes according to the rotation angle of the motor.

At first, this mechanism was controlled by a single board computer with several sensors. The tail servomotor rotates at a constant speed, and the tail oscillates at a constant frequency with a sine wave. Then, the swimming speed $\mathrm{Vx}$ is detected by the water flow sensor, and the tail oscillating speed $\mathrm{Vy}$ is detected by the rotary encoder attached to the fulcrum of the tail drive mechanism. These signals are transferred to the I/O of single board computer, and the water input angle $\theta \mathrm{w}$ 
is calculated. From this value, the caudal fin angle $\theta \mathrm{f}$ is determined, and the caudal fin control servomotor operates to realize the angle. This flow is shown in Fig.2.

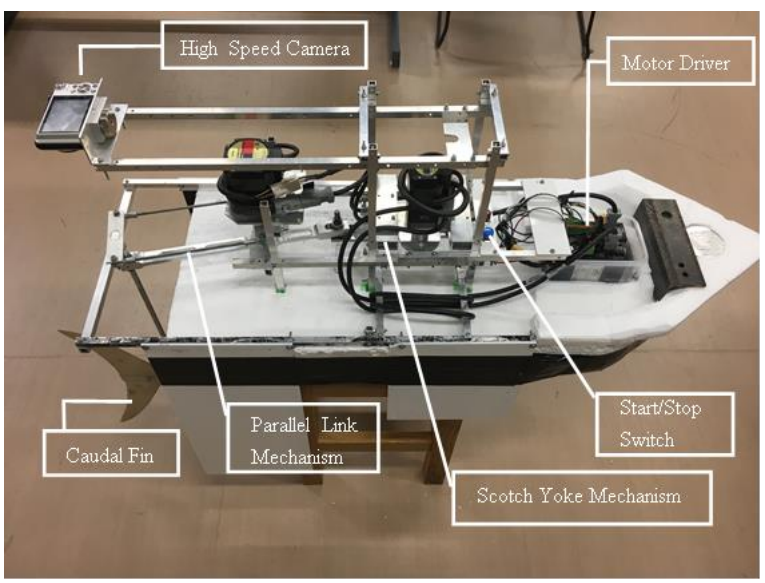

Fig.1 Overview of Fish Type Robot

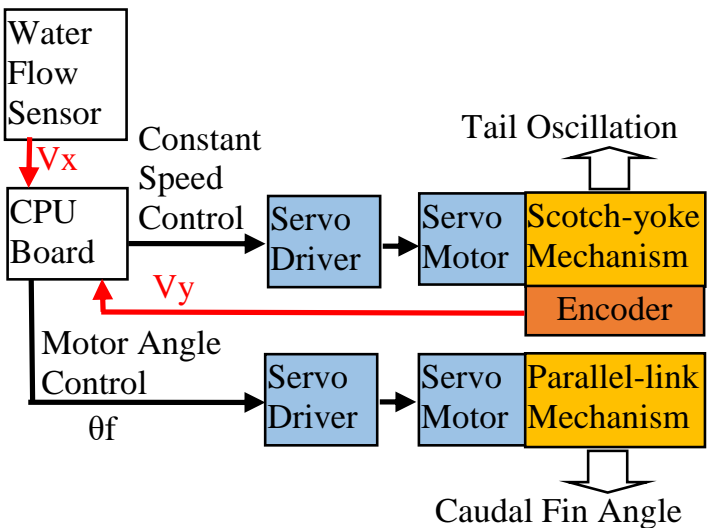

Fig.2 Original Controlling System

However, it has been found that this system has fatal problems to produce the desired swimming motion. As the frequency response of the servomotor is not sufficient, the motion angle of the caudal fin decreases and the phase is greatly delayed. In addition, CPU sensing and calculation time will cause phase lag of caudal fin motion. Fig. 3 shows the measured frequency response from the motor angle control signal (analog voltage) to the caudal fin angle (measured using a potentiometer).

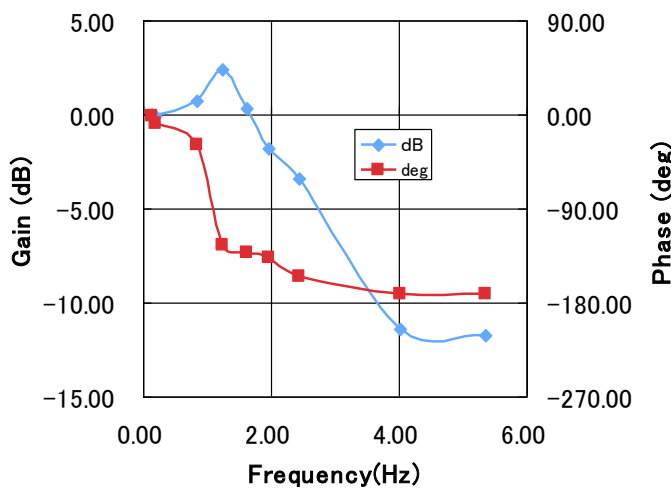

Fig.3 Frequency Response of Caudal Fin Angle
Therefore, we adopted a stepping motor instead of a servomotor. Modern stepping motors have excellent frequency characteristics. Therefore, there is no angle reduction or phase lag. In addition, the water flow sensor and CPU have been eliminated, and a device capable of setting the motor operation waveform in the stepping motor driver has been adopted. The two stepping motors that drive the tail and caudal fin start at the same time, so that synchronized motion can be realized. This system is shown in Fig.4.

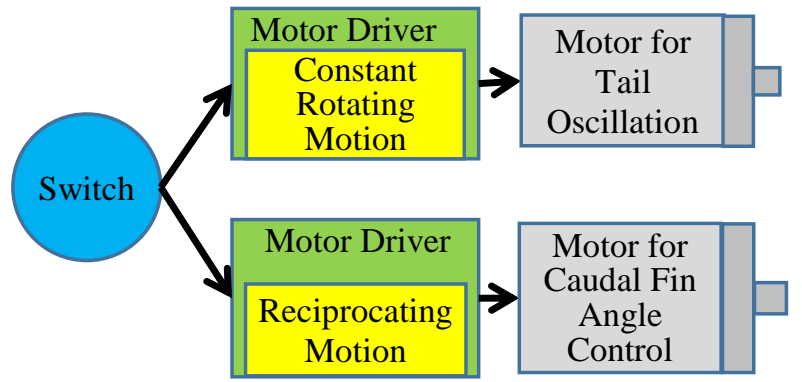

Fig.4 Synchronized Motion Control of Tail and Caudal Fin

Because the motor angle control signal is digital, it is not possible to measure frequency response data using analog measurement equipment. Therefore, We shot a video of the oscillation of the tail and the angle of the caudal fin with a high-speed camera and confirmed the response. Some shots of the video are shown in Fig.5.

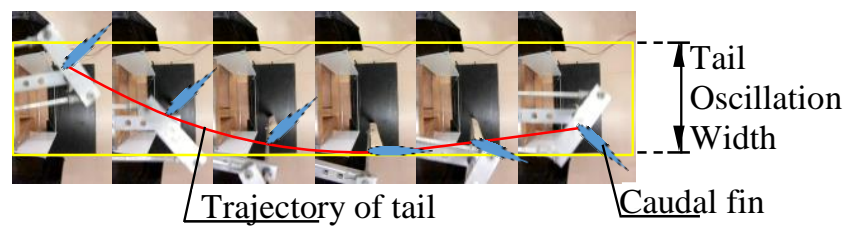

Fig.5 Confirmation of Motion Response

The caudal fin used for this mechanism adopted a rectangular caudal fin to compare experimental results and analytical knowledge as shown in Fig.6.

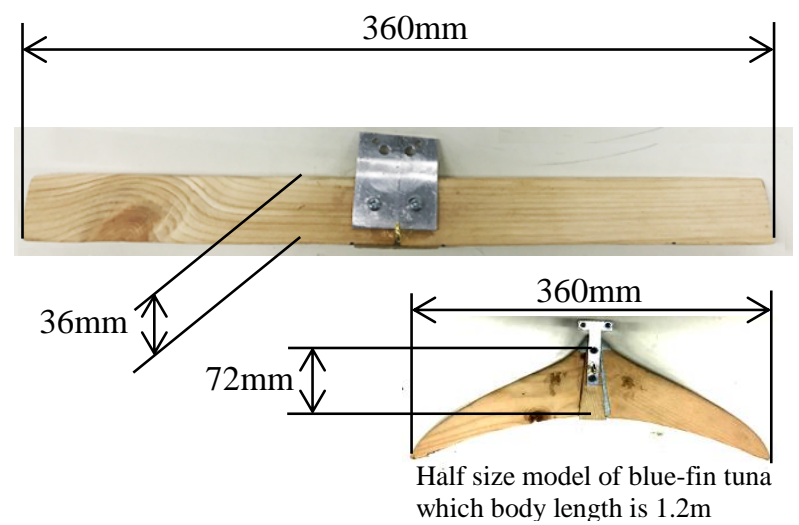

Fig.6 Appearance and Size of Caudal Fin

The lower right picture in Fig. 6 is the 1/2-scale caudal fin model of a $1.2 \mathrm{~m}$-long bluefin tuna. The rectangular caudal fin is made to have the same wing length and the 
same wing area for the tuna caudal fin model. The crosssectional shape of the both caudal fin model was manufactured to be the same as the cross-sectional shape of the actual bluefin caudal fin shown in Fig.7. The lift and drag of both caudal fins were measured for each angle of attack. The results are shown in Fig.8.

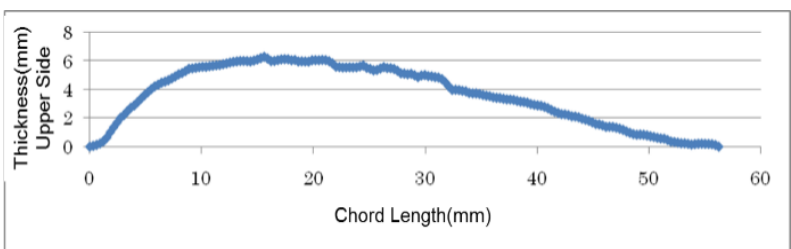

Fig.7 Cross-sectional Shape of the Caudal Fin of Bluefin Tuna

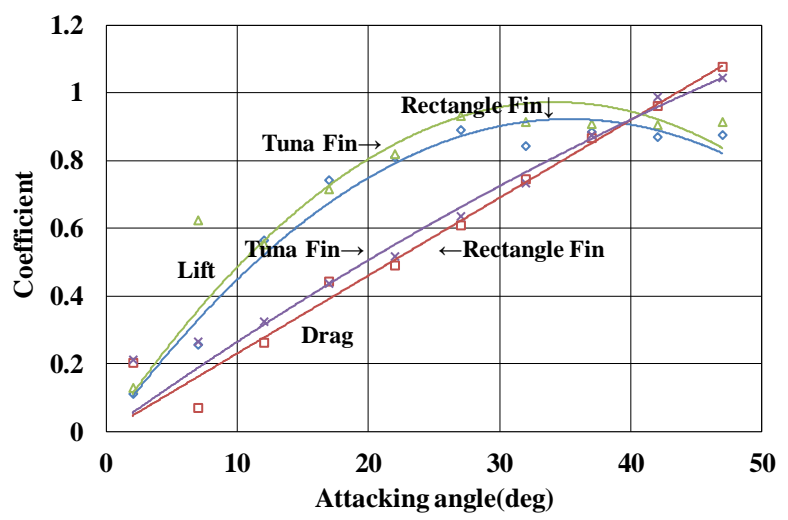

Fig.8 Lift and Drag Force Coefficient of Tuna and Rectangular Caudal Fin.

Thus, we developed the tuna type robot as shown in Fig.1. As you can see in Fig.1, we don't finish the water proof system of this robot yet, so this robot mechanism and control system are mounted on the ship shaped float and aligned only the caudal fin to sink into the water. The length of ship shaped float is $0.6 \mathrm{~m}$. Swimming speed of this ship is measured by taking movie which catches the tuna type robot and tape measure at the same time as shown in Fig.9.

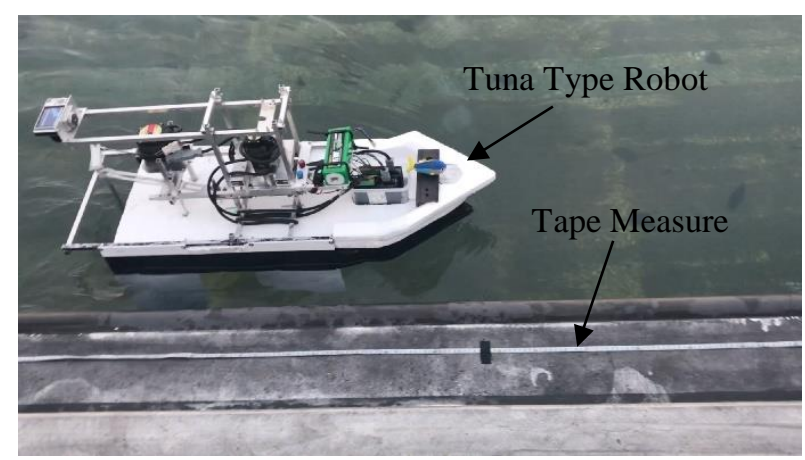

Fig.9 Measuring the Speed of Tuna Type Robot

\section{B. Caudal Fin Motion Scheme by Feathering Parameter}

Nakashima and Ono [3] determine the caudal fin motion as shown in Fig.10.

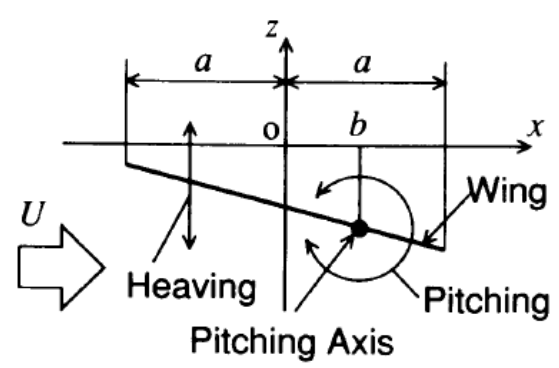

Fig.10 Determination of Caudal Fin Motion (Quote from [3])

Where

$U:$ Swimming Speed

$\omega$ : Tail Heaving Frequency

$h$ : Tail Heaving Width

$\alpha$ : Caudal Fin Pitching Width

$b$ : Caudal Fin Pitching Axle Position

$2 a$ : Caudal Fin Chord

The motion is separated to heaving motion and pitching motion. Heaving motion is determined as parallel moving to the vertical direction $\mathrm{Z}$ toward the water flow U. Pitching motion is determined as rotation around " $b$ " fulcrum. Nakashima and Ono [3] determine the caudal fin motion as Eq. (1).

$$
z=h \cos \omega t+\alpha(x-b) \sin \omega t
$$

In this equation, the phase difference between heaving and pitching is fixed at 90 degrees. But, the changing of " $b$ " position corresponds to the changing of both motion phase, So, Eq. (1) can express every motion of the caudal fin. Here, the feathering parameter $\theta$ is determined as Eq. (2).

$$
\theta=\frac{U \alpha}{\omega h}
$$

This parameter indicates the amplitude ratio between heaving and pitching. Also, $\theta$ is a dimensionless value. Nakashima and Ono's report [3] says $\theta=0.8$ motion brings the most effective swimming ability.

\section{Caudal Fin Motion Scheme by Wing Theory}

As the blue-fin tuna swims, the caudal fin moves to forward at constant speed with oscillation. This trajectory can be described as a sine waveform. The angle of inclination of this sine wave in the direction of swimming is the incoming water angle to the caudal fin. It is expressed as $\theta \mathrm{w}$. In order to generate propulsion using the lift of the caudal fin, the inclination angle $\theta$ f of the caudal fin must be smaller than the incoming water angle $\theta \mathrm{w}$. Therefore, we proposed the $\theta \mathrm{f} / \theta \mathrm{w}=$ constant $(<1)$ as the operating rule of the caudal fin. The principle of generating the propulsive force Fx using the lift $\mathrm{L}$ is shown in Fig.11. The kinematic relationships between each parameter are shown in Eq. (3) to (9). Where Vx is swimming speed, and Vy is tail oscillation speed. 


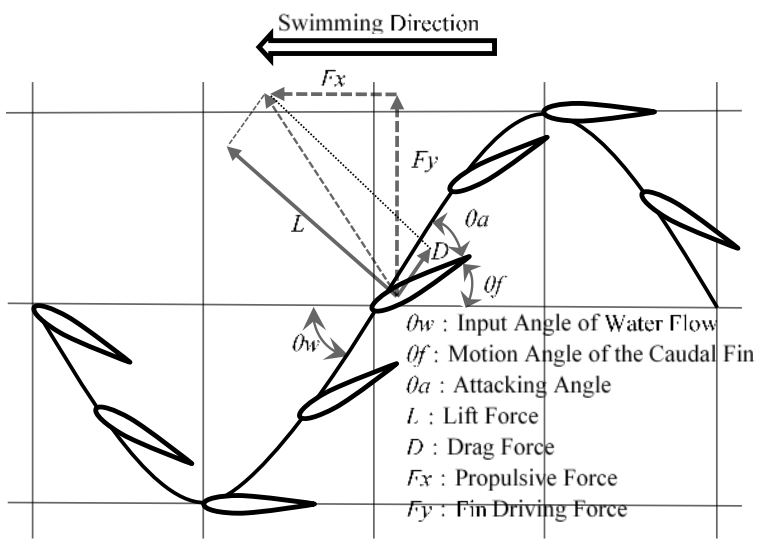

Fig.11 Caudal Fin Angle toward Water Flow to generate Propulsive Force

$$
\begin{aligned}
& \theta w=\operatorname{atan} 2\left(\frac{V y}{V x}\right) \\
& V=\sqrt{V x^{2}+V y^{2}} \\
& \theta a=\theta w-\theta f \\
& D=\mathrm{fd}(V, \theta a) \\
& L=\mathrm{fl}(V, \theta a) \\
& F x=-L \sin \theta w+D \cos \theta w \\
& F y=L \cos \theta w+D \sin \theta w
\end{aligned}
$$

Here, the functions $\mathrm{fl}$ and $\mathrm{fd}$ are based on the data obtained by measurement shown in Fig.8. Equations (3) to (9) were calculated by changing the parameter $\theta \mathrm{f} / \theta \mathrm{w}$ under the conditions of swimming speed of $V x=2.0 \mathrm{~m} / \mathrm{s}$ and tail oscillation frequency of $5 \mathrm{~Hz}$. And the result of having calculated average value Fx of driving force, and average value Fy of driving force is shown in Fig.12. From this, it can be seen that $\theta \mathrm{f} / \theta \mathrm{w}=0.85$ is the most effective.

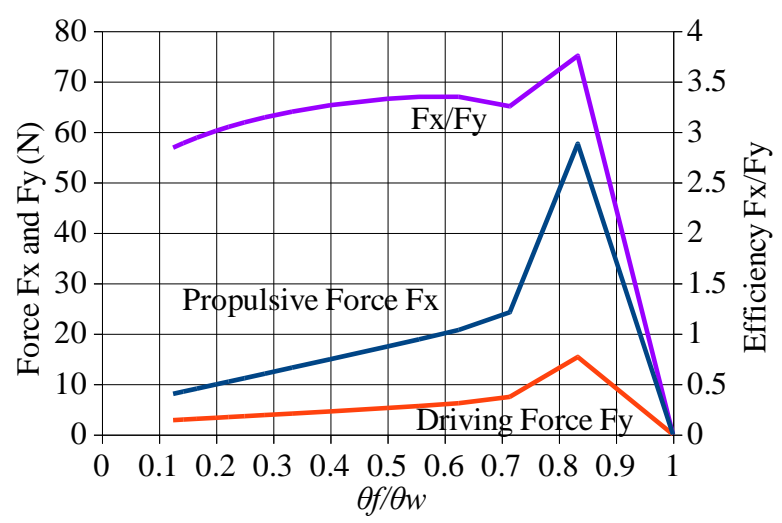

Fig.12 Propulsive Efficiency toward $\theta \mathrm{f} / \theta \mathrm{w}$

\section{RESULTS}

\section{A. Setting of operation data to motor driver}

We prepared caudal fin angle operation data with the following parameters for tail oscillation $2 \mathrm{~Hz}$ and $5 \mathrm{~Hz}$.

$\theta=0.3,0.4,0.5,0.6,0.7,0.8,0.9$

$\theta f / \theta w=0.35,0.45,0.55,0.65,0.75,0.85,0.9$ (11)
These motion data consist of angle data and speed data. As an example, Fig. 13 shows data of $\theta=0.4$ and 0.8 when the tail oscillates at $5 \mathrm{~Hz}$, and Fig.14 shows data of $\theta \mathrm{f} / \theta \mathrm{w}=0.45$ and 0.85 .
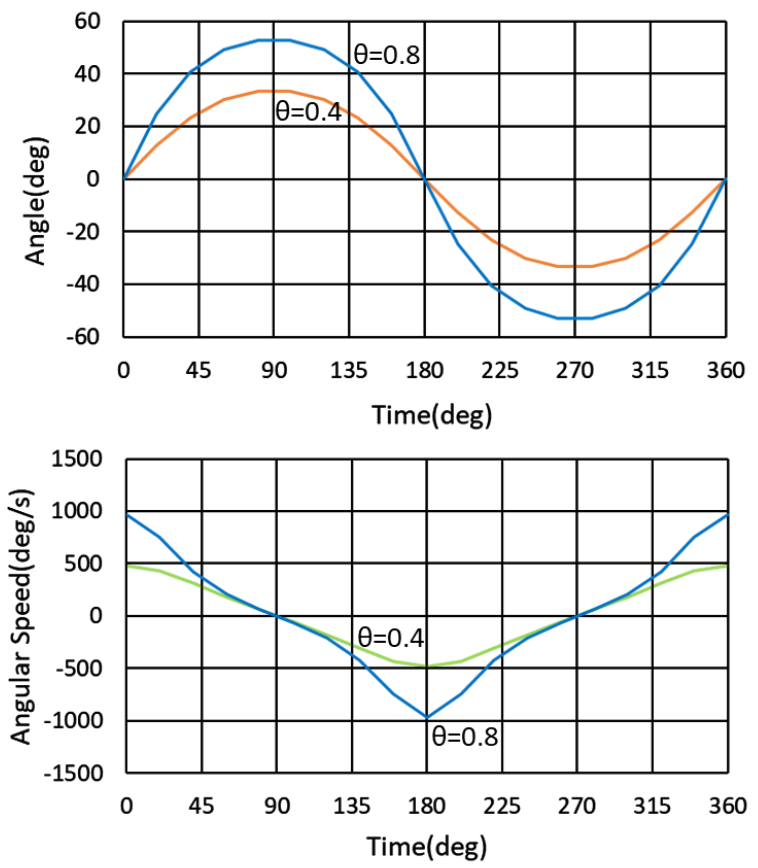

Fig.13 Caudal Fin Angle and Speed Pattern with $\theta=0.4 \& 0.8$ in $5 \mathrm{~Hz}$ Tail Oscillation
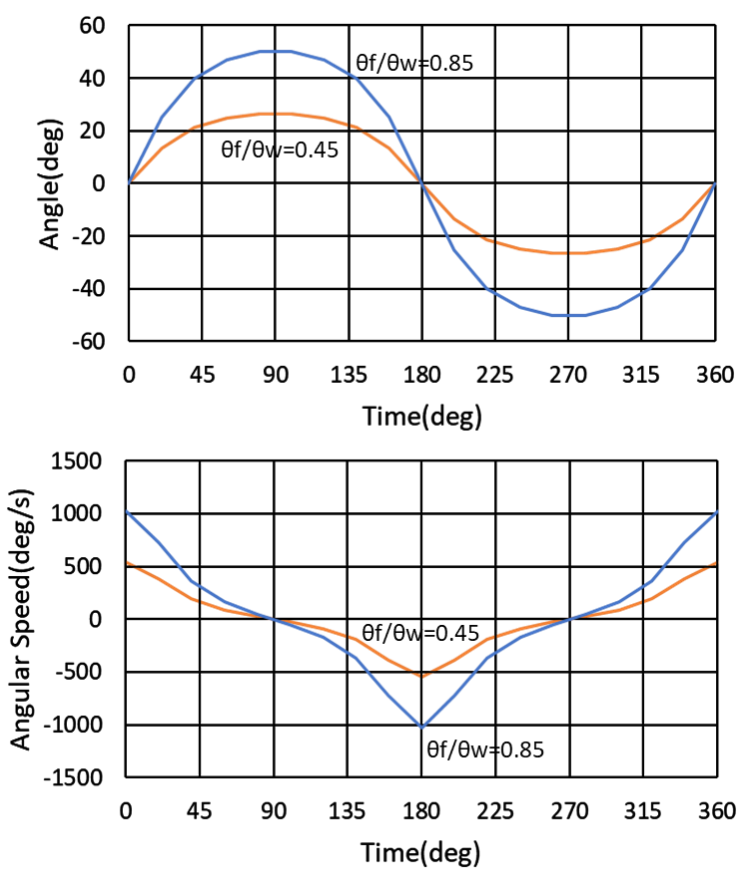

Fig.14 Caudal Fin Angle and Speed Pattern with $\theta f / \theta w$ $=0.45 \& 0.85$ in $5 \mathrm{~Hz}$ Tail Oscillation

\section{B. Swimming Speed}

The caudal fin angle was controlled by installing the data shown above in the motor driver, and the 
swimming speed was measured by the method shown in Fig.9. Fig.15 shows the relationship between the $\theta$ or $\theta \mathrm{f} / \theta \mathrm{w}$ values and the swimming speeds at the tail oscillation of $2 \mathrm{~Hz}$. This figure plots the results of three experiments. Since the experiments are conducted using an outdoor pool, it is thought that there is a variation due to the influence of wind.
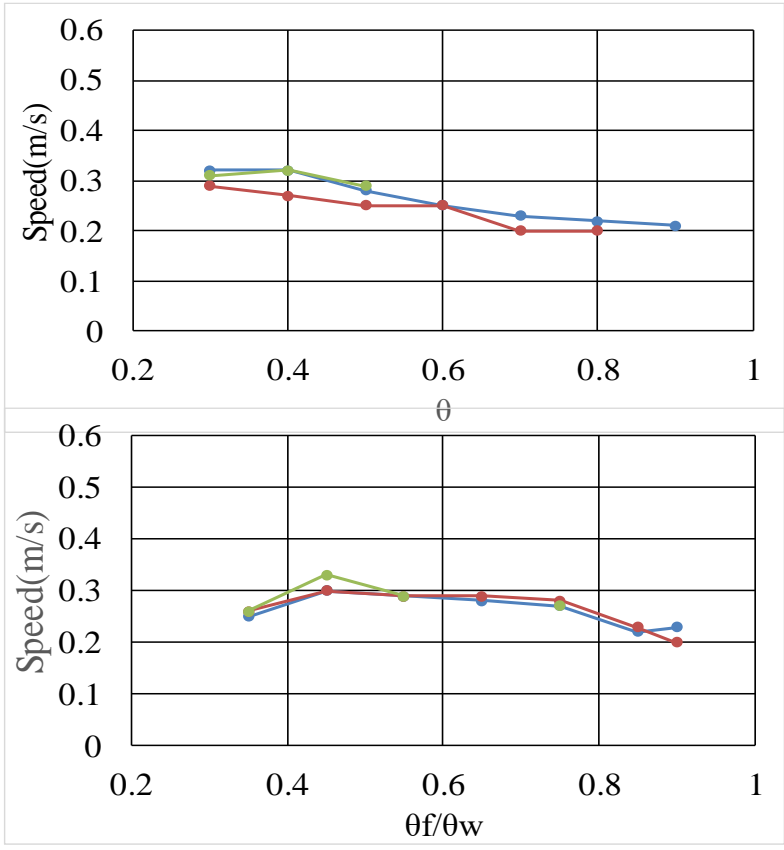

Fig.15 Relationship between $\theta$ or $\theta f / \theta w$ Value and Swimming Speed at $2 \mathrm{~Hz}$ Tail Oscillation

And Fig.16 shows the relationship between the $\theta$ or $\theta \mathrm{f} / \theta \mathrm{w}$ value and the swimming speed at the tail oscillation of $5 \mathrm{~Hz}$. This figure plots the results of two experiments.

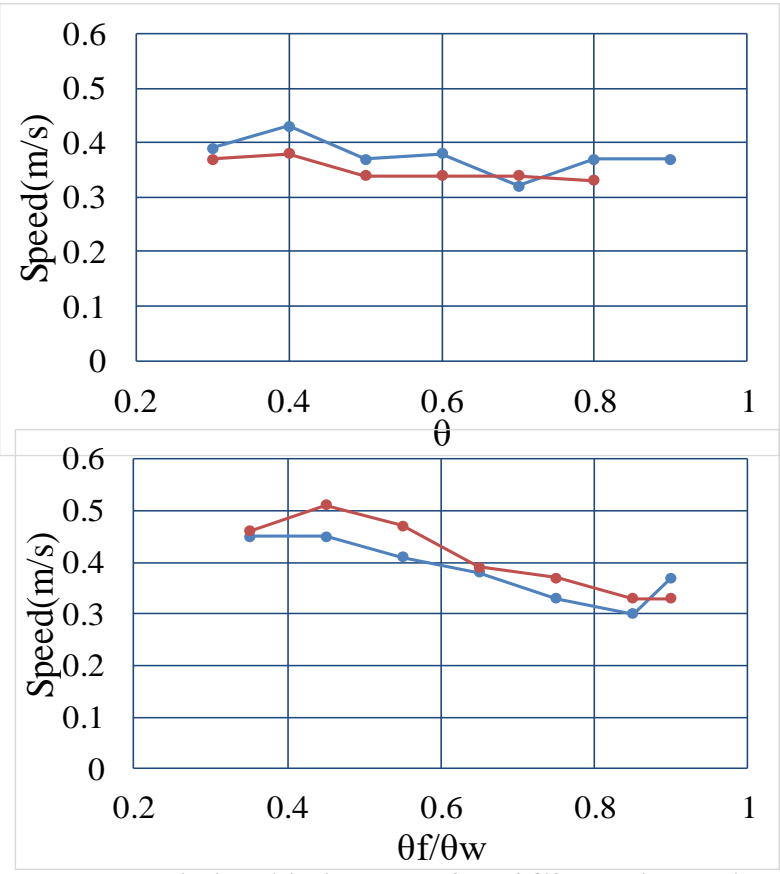

Fig.16 Relationship between $\theta$ or $\theta \mathrm{f} / \theta \mathrm{w}$ Value and Swimming Speed at $5 \mathrm{~Hz}$ Tail Oscillation.

\section{DISCUSSION}

\section{A. Comparison of theoretical analysis and experiment}

According to theoretical analysis, caudal fin motion at $\theta=0.8$ or $\theta f / \theta w=0.85$ seems to produce the most effective propulsion. However, experimental results show that motion at $\theta=0.4$ or $\theta \mathrm{f} / \theta \mathrm{w}=0.45$ provides the fastest speed. In order to investigate the cause of this result, according to the data in Fig.15, the numerical calculation by wing theory was performed again at a swimming speed of $0.3 \mathrm{~m} / \mathrm{s}$. The results are shown in Fig. 17 .

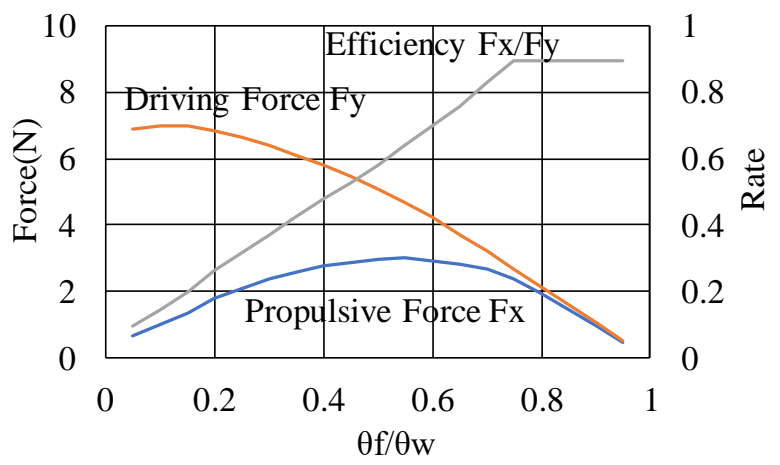

Fig.17 Propulsive Efficiency toward $\theta \mathrm{f} / \theta \mathrm{w}$ at Swimming Speed $0.3 \mathrm{~m} / \mathrm{s}$ by using Wing Theory

The propulsion efficiency shows the highest value around $\theta \mathrm{f} / \theta \mathrm{w}=0.75$. However, the driving force $\mathrm{Fx}$ becomes highest around $\theta \mathrm{f} / \theta \mathrm{w}=0.55$. This shows a tendency similar to the experimental results. To confirm this argument, we calculated the attack angle at a swimming speed of $0.3 \mathrm{~m} / \mathrm{s}$, a tail oscillation frequency of $2 \mathrm{~Hz}$, and a $\theta \mathrm{f} / \theta \mathrm{w}$ value of 0.55 or 0.75 . The results are shown in Fig. 18.

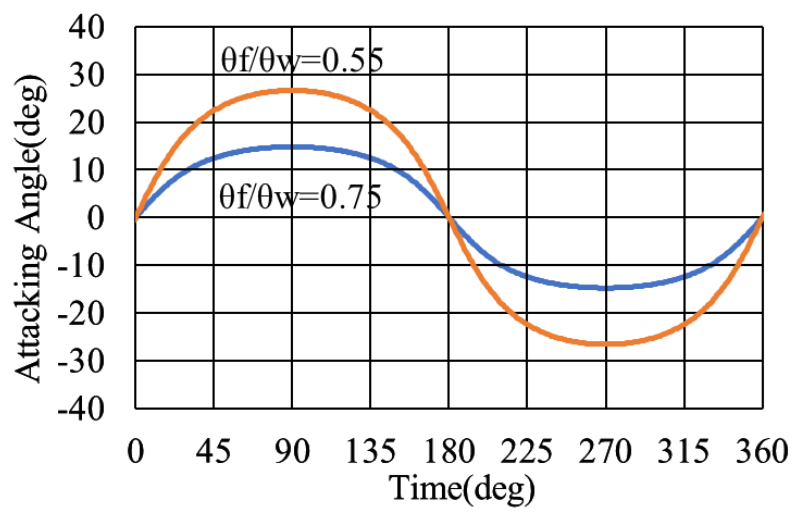

Fig.18 Attack Angle of Caudal Fin toward Water Flow. (Swimming Speed $=0.3 \mathrm{~m} / \mathrm{s}$, Tail Oscillation Frequency

$$
=2 \mathrm{~Hz} \text { ) }
$$

Looking at Fig.18 and Fig.8, it can be seen that the motion of $\theta \mathrm{f} / \theta \mathrm{w}=0.55$ uses the attack angle that generates the highest lift. However, in this case, the drag also increases, and the driving power of the motor that drives the tail is considered to be high. Therefore, it is 
thought that the propulsion efficiency will not be high. Therefore, it is necessary to measure the motor current in the near future. In the same sense, it is assumed that the operation of the feathering parameter $\theta=0.4$ produces the highest propulsion force. However, since the driving force is also considerably increased, the efficiency is considered not to be high. In addition, although the values of $\theta \mathrm{f} / \theta \mathrm{w}$ showing the maximum propulsive force are slightly different between numerical calculation and experiment, the reason should be considered in the future. At this point, it can be said that because the tail fin is held cantileverly in the structure of the fish robot shown in Fig.1, the shaft may be slightly bent during oscillating.

\section{B. Comparison between Blue-fin Tuna and Tuna Type Robot}

It is necessary to consider the dimensionless frequency as an index to evaluate the swimming power by the caudal fin. Here, instead of the dimensionless frequency, it was evaluated by the migration number $\mathrm{Sw}$, which is its reciprocal. As a representative, $\mathrm{Sw}$ can be obtained from the swimming speed when the tail is oscillating at 2Hz, as shown in Fig. 19.
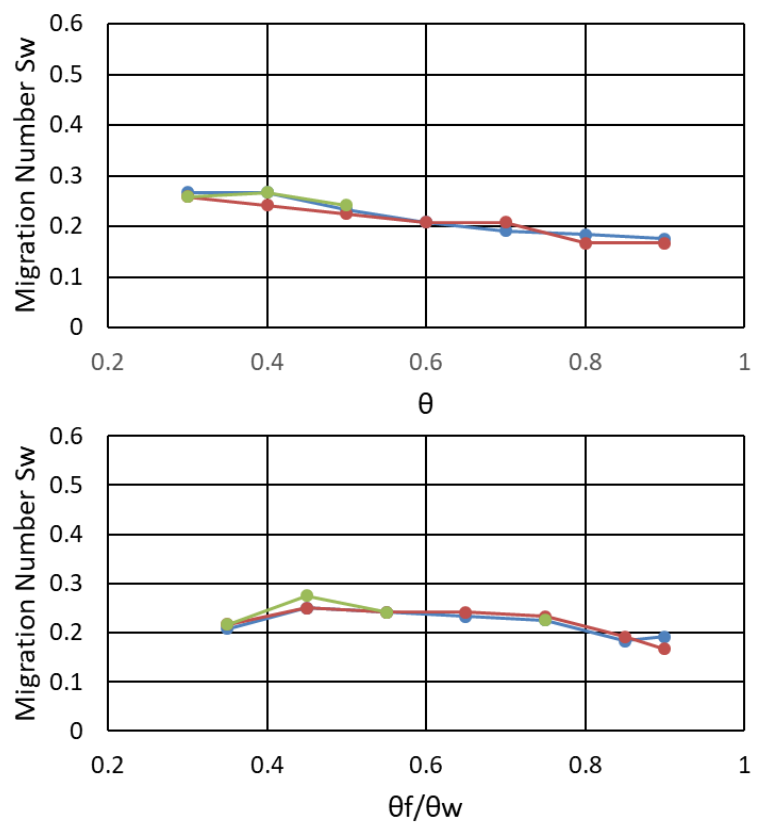

Fig.19 The Migration Number Sw toward $\theta$ or $\theta f / \theta w$

The migration number $\mathrm{Sw}$ indicates the ratio of swimming length to body length during one beat of tail oscillation. Bainbridge and Nagai have found that almost all kinds of fish swim at a speed of $\mathrm{Sw}=0.6$ [7]. We can see that the swimming ability of the fish type robot we developed is less than half that of fish at $\theta \mathrm{f} / \theta \mathrm{w}=0.45$ at most.

$$
\mathrm{Sw}=\frac{0.33(\mathrm{~m} / \mathrm{s})}{2(\mathrm{~Hz}) \times 0.6(\mathrm{~m})}=0.275
$$

Meanwhile, Bainbridge also found that the ratio of tail oscillation width to body length is nearly constant at 0.2 for almost all fish. However, this ratio of our fish type robot is only 0.13 . This is a design mistake of our fish type robot. Therefore, we decided to evaluate the swimming ability using the oscillation width reference migration number Swb. The Swb value of fish is as follows.

$$
\mathrm{Swb}=\frac{\mathrm{Sw}}{0.2}=\frac{0.6}{0.2}=3.0
$$

The Swb value of our tuna type robot becomes as follows.

$$
\mathrm{Swb}=\frac{\mathrm{Sw}}{0.13}=\frac{0.275}{0.13}=2.1
$$

So, the swimming ability of tuna type robot seems to be $70 \%(=2.1 / 3.0)$ of fish. Of course, the drag of the fish robot itself seems to be greater than that of real fish. So, we have to compare the drag in the following study.

\section{CONCLUSIONS}

Our fish type robot was found to swim at a speed of about $0.3-0.5 \mathrm{~m} / \mathrm{s}$. From experimental results and theoretical verification, in this speed range, the driving force by the caudal fin is maximum at $\theta=0.4$ or $\theta \mathrm{f} / \theta \mathrm{w}=0.45$. However, the propulsion efficiency has not been evaluated yet. So, we have to prepare a measurement system for the driving force.

The experimental results show that the fish type robot in this study has $70 \%$ swimming ability to the real fish in the meaning of the oscillation width reference migration number Swb. In order to improve the swimming ability, it is necessary to increase the ratio of the tail oscillation width to the body length. In addition, it is necessary to reduce the drag of the robot body.

If our fish type robot can swim faster than ever, for example $2 \mathrm{~m} / \mathrm{s}$, we need to reconfirm the relationship between swimming motion and swimming speed according to the report of Nakashima and Ono [3].

\section{ACKNOWLEDGEMENTS}

We appreciate to Tsutsujigaoka primary school for let us use swimming pool, especially vice principal Yamamoto.

And we must also thank Mr. Migimatsu and Mr. Amano who are the engineer of our college by advising toward designing, machining, assembling.

\section{REFERENCES}

[1] Kato, N. et al., Aero Aqua Bio-Mechanics, Study Group of Aero Aqua Bio-Mechanisms (2010)

[2] Azuma, A., The Bio kinetics of Flying and Swimming, Springer-Verlag Tokyo (1992) 
[3] Nakashima, M. and Ono, K., Dynamics of Two-Joint Dolphinlike Propulsion Mechanism (1st Report, Analytical Model and Analysis Method), Journal of the Japan Society of Mechanical Engineers (Series B), Volume62, Issue600, pp.136-143(in Japanese) (1996)

[4] Nakashima, M. and Ono, K., Dynamics of Two-Joint Dolphinlike Propulsion Mechanism (2nd Report, Optimum Motion for Primary Body Form), Journal of the Japan Society of Mechanical Engineers (Series B), Volume62, Issue602, pp.67-74 (in Japanese) (1996)

[5] Kugai, K. and Gomyouta, Y., Analysis of Swimming Ability of Blue Fin Tuna, Research Reports of Kindai University Technical College, No.2, pp.11-16(in Japanese) (2008)

[6] Kugai, K. and Hamaguchi, R., Development of Fish type Robot based on the Analysis of Swimming Motion of Blue Fin Tuna, Research Reports of Kindai University Technical College, No.3, pp.13-17(in Japanese) (2009)

[7] Tanaka, I. and Nagai, M., Resistance and propulsion hydrodynamics (Learn from fast swimming ability of aquatic organisms), Ship and Ocean Foundation (in Japanese) (1996) 